\title{
The Topology Optimization for Shape Finding of Plane Stress and Strain Problems
}

\author{
Kanggeun Park \\ I'ST Institute of Technology, Seoul, South Korea \\ Taehong Ha \\ Korea Engineering Consulting Co. Ltd., Seoul, South Korea \\ BoongKyo Seo \\ Corresponding Author, Department of Architecture, \\ Gachon University, Seongnam, South Korea
}

\begin{abstract}
The optimization design are classified into section, structural and topology optimization. The section optimization is analyzed for initially assumed sectional dimension, and based on limiting values on stresses and displacements. The structural optimization is appropriately resizing variables in order to find an optimum condition with the minimum possible material volume, mass or weight. The topology optimization is a mathematical method that optimizes material layout so that satisfies a given design condition, boundary conditions and constraints. The topology optimization has wide applications for aerospace, mechanical and civil engineering. The 3D printer technology can be used to topology optimization when designing new products. The objective of this study is to analyze the shape results of topology optimization for arbitrary models within plane stresses and strain problems by NISA Software. The application of the topology optimization in various fields of engineering may significantly improve design cost and quality. The authors would like spread the concept and ideas of topology optimization.
\end{abstract}

Keywords - Optimization Design, Section Optimization, Structural Optimization, Topology Optimization

\section{INTRODUCTION}

The optimization design is useful tools to predict an optimum component design in early design process. The optimization techniques are becoming increasingly important to satisfy complicated and detailed engineering requirements. Recently the engineers have realized that topology optimization has become essentially for the design of structural elements. The topology optimization is to find the optimal path of material distribution. Comparing to size and shape optimization, topology optimization is to determine the reasonable structure for the conceptual design of new products and improvement design for existing products. Structural topology optimization is to find the premise material distribution scheme under the design requirements to specify the design direction. Topology optimization design is a computational method for achieving optimal material distribution without knowing the shape of the structural members. The topology optimization can be used to develop the shape of new structure. The history of the topology optimization back to the truss theory proposed by Michell in 1904 [1]. In 1960, Schmit [2] adopted mathematical programming theory to solve optimization problems of elastic structure under the condition of multiple loads. Topology optimization theory of continuum structure has developed rapidly. Many topology optimization methods including variable thickness method, variable density method, independent continuum mapping method, and nodal density method [3] have been proposed. The topology optimization model of 3D continuum structure with objective function of minimum compliance and design variable of material distribution is established [4]. This study is to analyze the shape results of topology optimization for arbitrary models in plane stresses and strain problems by using NISA 2016 Software. The topology optimization can be widely applied to the mechanical and structural engineering, the 3D printer technology can be used for designing new products.

\section{THEORETICAL OVERVIEW OF TOPOLOGY OPTIMIZATION DESIGN}

\section{A. Theoretical Overview of Optimization Design}

In the design of modern structures, weight reduction with structural performance is an important requirement. Most of the investigations focus on the dimension, shape and topology optimization. Section optimization find a dimension of a section of any specified that minimize its area of cross section with constraints of section properties and other related constraints. Find a design variable vector that minimized the cost function. In the typical sizing problem the goal may be to find the optimal thickness distribution or the optimal member area. The optimal 
thickness distribution minimizes a physical quantity such as the mean compliance, maximum stress, deflection, etc. The shape optimization is to find the optimum shape which satisfies the design variable. Topology optimization is to find the optimal lay-out of a structure within a specified region for the features such as the number, location, shape and connectivity of domain. The general optimal shape design is formulated as a material distribution problem. The optimal method allows for an efficient prediction of the optimal topology, the optimal shape and the optimal use of the prescribed possible conditions. The methodology has become a fairly widespread tool in the industrial applications, the commercial software is very useful for the applications of topology design method in practice. For the efficient use of topology design, the problems should be formulated on a reference domain that is chosen as simple as possible to reduce the size of the analysis problems. The use of automatic mesh generator will simplify the complicated problems $[1,2,3,4,5]$.

\section{B. Procedure of Topology Optimization Design}

The problem of finding the optimal topology by the material distribution method involves finding the optimal distribution of material described by the density and the displacement field of the optimal design. The topology optimization used the Lagrange multiplier formulation to generate what are generally known as the optimality criteria expressions. Constraint on structural response quantities, such as nodal displacements and element stresses, can be written in the form to set up these expressions by means of sensitivity analysis. In the topology optimization the method decides on whether to update and resolve the optimality criteria problems, or to accept the design the design and continue into the next stage of optimization. Several steps of updating and redesign may be necessary at this stage. All shape changes in this stage are obtained by discretizing the solution into $0-1$ decisions and appropriately removing elements. The next stage involves a series of intermediate design based on a sensitivity approach. The intermediate designs are aimed at increasing the efficiency of the design to try and insure, the optimum design will be a subset of the most efficient design obtained in this stage. The measure of efficiency is the virtual volume obtained as the ratio of material volume to the most critical factor to the constraint surface. In this stage some of removed elements are restored to the structure based on the results of sensitivity analysis. The iterative intermediate design stage ends when the program selects one of the generated designs as the most efficient design. The next design step is a repetition of the outlined procedure, starting within this most efficient design from the last design step. The optimization process will continue until the specified total number of iterations or design steps are satisfied. Execution will end earlier if the program is not able to improve upon the last most efficient design generated. If the number of iterations prescribed is founded to be insufficient after the process ends, it is possible to restart the process from the last most efficient design founded by the program [6].

One of the important features is that no boundary parameterization of the structure is required for shape optimization. With boundary parameterization, even relatively small design changes may create substantial distortion of elements, necessitating frequent re-meshing of the model. The model is designed to optimize structures modeled by a fine mesh of small and simple elements. Thus, over a given region, the combined effect of these small elements is that region simulates a larger more sophisticated element, with an important advantage in terms of shape optimization. The element shape manipulation is simplified into decisions regarding the conservation or deletion of elements. With this approach, very large design changes may be accomplished without the need for any refinement of the finite element mesh, while always retaining elements with excellent aspect ratios. On the other hand, the generated designs may be expected to have a surface roughness of the order of the size of an element. It is recommended that an application design can be created to base the optimum shape, and modeled with more sophisticated finite elements and re-analyzed. The optimization analysis may stop execution not because the optimum design has been reached, but because it is not possible to proceed any further with the remaining finite element mesh. If further optimization is required, the latest most efficient design should be conservatively re-modeled with a refined finite element mesh and re-submitted for shape optimization. There will be some difference between the computed responses for the refined and unrefined designs with the response for the refined mesh being more accurate. The analysis is not able to add material beyond the set presented by the initial design, it is good practice to start out with a feasible design that is believed to encompass the optimum design. In the first mode, material may be removed from anywhere in the structure. The first mode may be especially useful in determining a basic shape for a new system. It may be more appropriate to use the second mode of shape optimization which allows for shape variation only on the original boundaries without creating new boundaries. In the two major modes of shape optimization, engineers can have a choice regarding whether any breaks should be allowed to take place. The optimum design should be performed sensitivity analysis for stress and displacement response quantities. In sensitivity analysis, it can be determined which elements in the structure contribute most and just as importantly, least to the response quantity. And then one virtual load is generated for each relevant response quantity. The sensitivity analysis results would make browsing virtually impossible if the contribution from all the elements were listed for each response 
quantity, it is needed to the choice of specified elements with contributions [6].

\section{A. The Topology Optimum Design of a Panel}

\section{APPLICATIONS OF TOPOLOGY OPTIMIZATION METHOD}

The model is a panel with two simple supports. The panel is loaded in plane in the middle by a force $\mathrm{P}$ applied in the downward direction. The two simple supports at the ends restrict translation in the $\mathrm{X}$ and $\mathrm{Y}$ directions. For the given single load case, the structure is to be optimized for minimum material volume, with constraint on the von Mises equivalent stress everywhere in the system as well as a constraint on the Y-direction translation at the point loading. The shape optimization are to be used, allowing shape changes to take place anywhere in the system, also allowing possible breaks. The structure is modeled triangular plane stress elements. The Mises stress constraint is applied to all elements, the displacement constraint is applied to 2 nodes. The best design found at iteration 64 . Any kinks are due to lack of sufficient number of elements in final stages. The much more improvement can be obtained after smoothing out the shape and remodeling with smaller elements. The percent decrease in volume is $84.57 \%$.

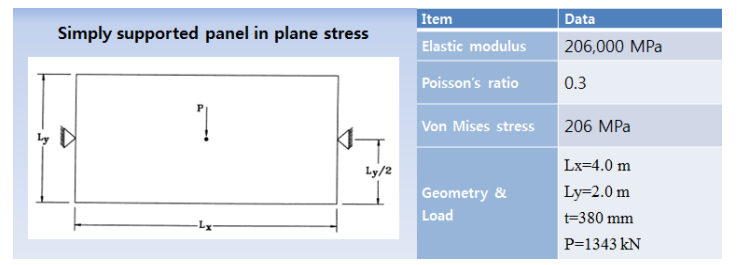

Figure 1. A panel with two simple supports at the end and a point load at the center

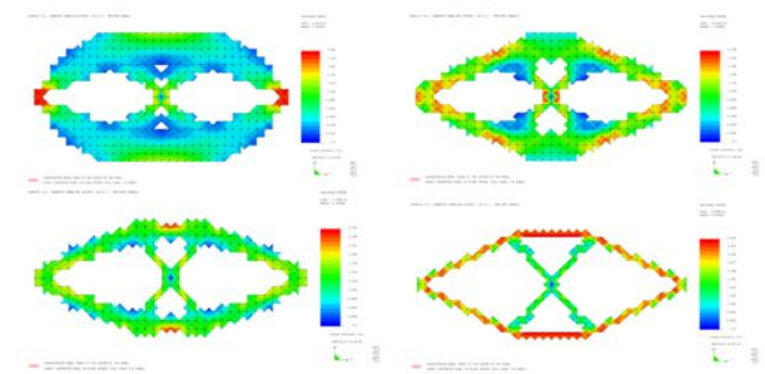

Figure 2. The topology optimum design of a panel with two simple supports

\section{B. The Topology Optimum Design of a Cantilever Panel}

The model is a cantilever panel in plane stress as shown in Figure 3. The load case is the distributed load acting as shown in the Figure 3. The structure is to be optimized for minimum material volume, the von Mises constraint is applied to all of the elements in the model. The panel is to be optimized for minimum material volume, with the shape variation limited only to the original boundaries and no breaks allowed. The best design founded at iteration 110. The most efficient design of design step 13 and 14 are very closed to the optimum design, but the best design obtained in iteration 110 . The percent decrease in volume is $56.74 \%$.

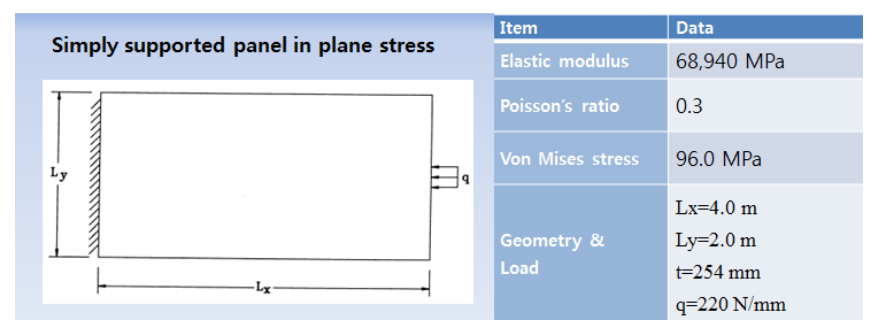

Figure 3. A cantilever panel applied to distributed load 


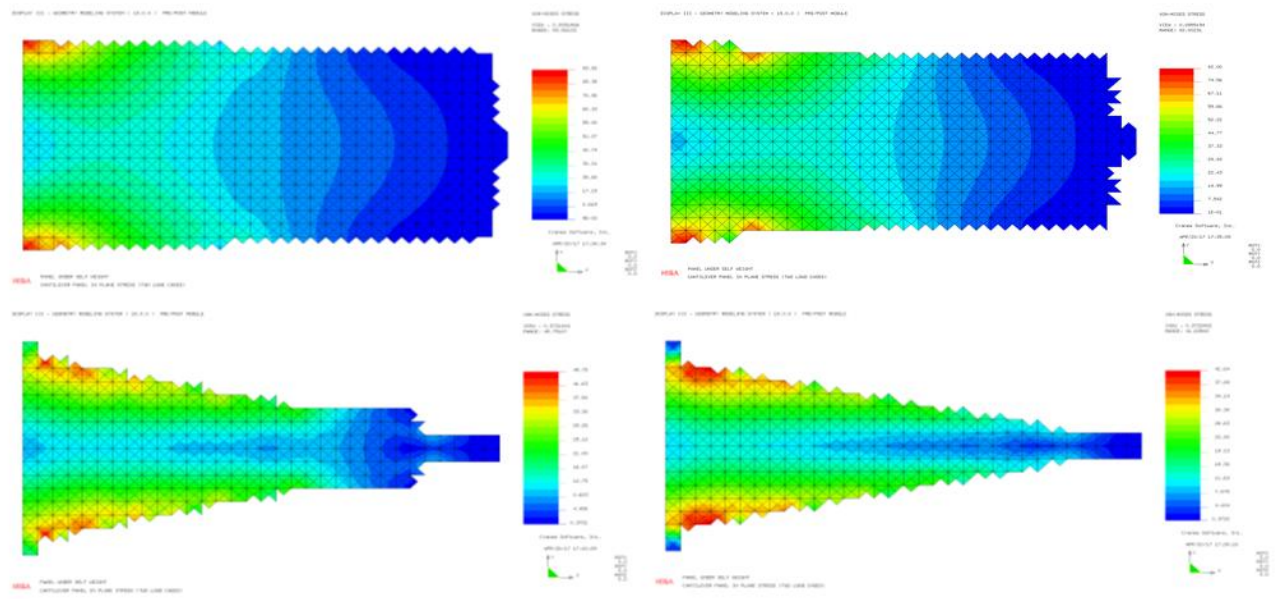

Figure 4. The topology optimum design of a panel with two simple supports

\section{The Topology Optimum Design of a Tunnel Cross-Section in Plane Strain}

The model is a fictitious tunnel cross-section. The loading condition is a uniform normal pressure on the outside perimeter of the structure on the sides and top. This normal pressure is considered to be hydrostatic in nature and is to follow the changing boundary as the structure is optimized. The shape of this tunnel is to be optimized for minimum total material cost with a limit on the maximum absolute value of the principal stresses. The shape variation is to be limited only to the original boundaries, any breaks will be allowed. The structure is modeled as triangular plane strain elements. The first rectangular layer of the elements at the bottom is frozen to prevent biasing of the support conditions. The constraint on the maximum absolute value of the principle stresses is applied to each of all elements. Started with the initial shape, this stage ended at the end of the first design step. The number 2 was founded to be a substantial improvement to the initial design, although it was still not feasible. In the some case, restarting the job with the results of iteration 2 does lead to feasible and eventually optimum shape is found. Stage 2 was started with the files obtained at iteration number 2 of stage 1 . The allowable number of iterations was increased. Although this stage was also started with an infeasible shape, a feasible shape was found at the end of design step1, and the program went on to find an optimal shape. The process terminated at iteration 134 (design step 11), the best design found at iteration 118 (design step 10). The most efficient design in the previous design step could not be improved upon during the intermediate design process. It is highly probable that the final shape is within close proximity of the global optimum, within the limitations of the remaining finite element mesh. The initial material cost 175 from stage 1 . The final material cost is obtained 84 . The percent decrease in volume is $52 \%$. The initial factor to constraint surface is 0.92569 , the final factor to constraint surface is 1.0006 . The change in material cost and design step number can be obtained. The smoothed shape is obtained from the final design.

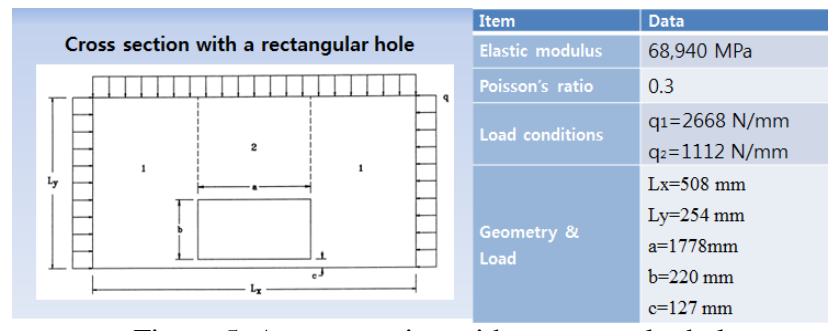

Figure 5. A cross section with a rectangular hole 


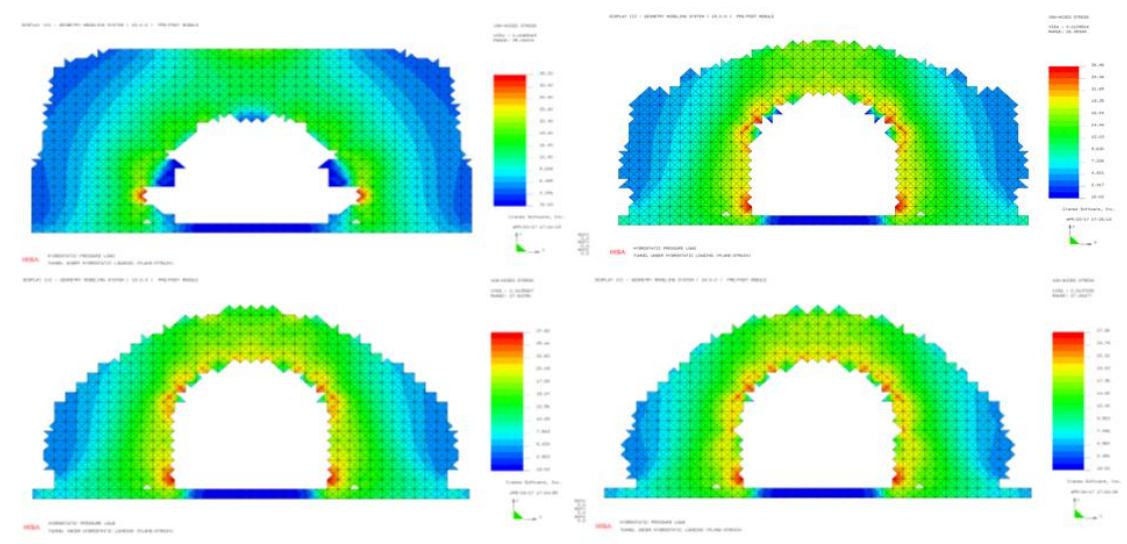

Figure 6. The topology optimum design of a cross section with a rectangular hole

\section{IV.CONCLUSION}

The optimization design is useful tools to predict an optimum component design in early design process, and to find the optimal path of material distribution. Comparing to size and shape optimization, topology optimization is to determine the reasonable structure for the conceptual design of new products and improvement design for existing products. Topology optimization design is a computational method for achieving optimal material distribution without knowing the shape of the structural members. Topology optimization is to find the optimal lay-out of a structure within a specified region, and the general optimal shape design is formulated as a material distribution problem. The optimal method is to for an efficient prediction of the optimal topology, the optimal shape and the optimal use of the prescribed conditions. Optimizations of material properties play an important role in the design of structures. Using more good material can give great payoffs for lower weight, higher stiffness and the improvement of structural response. The goal of designing efficient materials with prescribed elastic properties is defined as an optimization problem of minimization of material weight. Minimizing weight is considered to the reasonable design criteria because high stiffness to weight ratio is desirable in most industrial applications. The optimization problem can be solved by many different numerical optimization algorithms. In defining the optimality criteria algorithm, the mutual influence from element to element is ignored which means that the design variables are updated independently in each iteration step.

\section{ACKNOWLEDGEMENTS}

(a) This research was supported by a grant (17AUDP-B100343-03) from Architecture \& Urban Development Research Program funded by Ministry of Land, Infrastructure and Transport of Korean government.

\section{REFERENCE}

[1] Michell A G M., "The limit of economy of material in frame structures". Philosophical Magazine, 8(6), pp. 589-597, 1904

[2] Schmit L A., "Structural design by systematic synthesis," Proceedings of Electronic Computation, ASCE, 32(1), pp. 105-132, 1960

[3] Sigmund O, Maute K. "Topology optimization approaches", Structural \& Multidisciplinary Optimization, 48(6), 2013, pp. 1031-1055, 2013

[4] Bai Shiye and Zhu Jiejiang, "Topology Optimization Design of 3D Continuum Structure with Reserved Hole Based on Variable Density Method," Journal of Engineering Science and Technology Review 9 (2), pp.121-128, 2016

[5] NISA II manual, Cranesoftware, 2016 